\title{
Comparative transcriptome among Euscaphis konishii Hayata tissues and analysis of genes involved in flavonoid biosynthesis and accumulation
}

Wenxian Liang ${ }^{1,2}$, Lin $\mathrm{Ni}^{2,3}$, Rebeca Carballar-Lejarazú ${ }^{4}$, Xiaoxing Zou ${ }^{1,2}$, Weihong Sun ${ }^{1,2}$, Lingjiao Wu ${ }^{1,2}$, Xueyuan Yuan ${ }^{1,2}$, Yanling Mao ${ }^{2,5}$, Wei Huang ${ }^{2,6}$ and Shuangquan Zou ${ }^{1,2^{*}}$

\begin{abstract}
Bachground: Euscaphis konishii Hayata, a member of the Staphyleaceae Family, is a plant that has been widely used in Traditional Chinese Medicine and it has been the source for several types of flavonoids. To identify candidate genes involved in flavonoid biosynthesis and accumulation, we analyzed transcriptome data from three E. konishii tissues (leaf, branch and capsule) using Illumina Hiseq 2000 platform.

Results: A total of 91.7, 100.3 and 100.1 million clean reads were acquired for the leaf, branch and capsule, respectively; and 85,342 unigenes with a mean length of $893.60 \mathrm{bp}$ and N50 length of $1307 \mathrm{nt}$ were assembled using Trinity program. BLASTx analysis allowed to annotate 40,218 unigenes using public protein databases, including NR, KOG/COG/eggNOG, Swiss-Prot, KEGG and GO. A total of 14,291 (16.75\%) unigenes were assigned to 128 KEGG pathways, and 900 unigenes were annotated into 22 KEGG secondary metabolites, including flavonoid biosynthesis. The structure enzymes involved in flavonoid biosynthesis, such as phenylalanine ammonia lyase, cinnamate 4-hydroxylase, 4-coumarate CoA ligase, shikimate O-hydroxycinnamoyltransferase, coumaroylquinate 3'monooxygenase, caffeoyl-CoA O-methyltransferase, chalcone synthase, chalcone isomerase, flavanone 3hydroxylase, flavonoid 3'-hydroxylase, flavonoid 3',5'-hydroxylase, flavonolsynthese, dihydroflavonol 4-reductase, anthocyanidinreductase, leucoanthocyanidin dioxygenase, leucoanthocyanidin reductase, were identified in the transcriptome data, 40 UDP-glycosyltransferase (UGT), 122 Cytochrome P450 (CYP) and 25 O-methyltransferase (OMT) unigenes were also found. A total of 295 unigenes involved in flavonoid transport and 220 transcription factors (97 MYB, 84 bHLH and 39 WD40) were identified. Furthermore, their expression patterns among different tissues were analyzed by DESeq, the differentially expressed genes may play important roles in tissues-specific synthesis, accumulation and modification of flavonoids.
\end{abstract}

Conclusion: We present here the de novo transcriptome analysis of E. konishii and the identification of candidate genes involved in biosynthesis and accumulation of flavonoid. In general, these results are an important resource for further research on gene expression, genomic and functional genomics in E. konishii and other related species.

Keywords: Euscaphis konishii Hayata, Transcriptome, Gene expression, Flavonoid, Biosynthesis, Transport, Transcrip factor

\footnotetext{
* Correspondence: zou@fafu.edu.cn

${ }^{1}$ College of Forestry, Fujian Agriculture and Forestry University, Fuzhou, China

${ }^{2}$ Fujian Colleges and Universities Engineering Research Institute of

Conservation \& Utilization of Natural Bioresources, Fujian Agriculture and

Forestry University, Fuzhou, China

Full list of author information is available at the end of the article
}

(c) The Author(s). 2019 Open Access This article is distributed under the terms of the Creative Commons Attribution 4.0 International License (http://creativecommons.org/licenses/by/4.0/), which permits unrestricted use, distribution, and reproduction in any medium, provided you give appropriate credit to the original author(s) and the source, provide a link to the Creative Commons license, and indicate if changes were made. The Creative Commons Public Domain Dedication waiver (http://creativecommons.org/publicdomain/zero/1.0/) applies to the data made available in this article, unless otherwise stated. 


\section{Background}

The major active ingredients of medicinal plants are secondary metabolites, which their biosynthesis and accumulation are different among development stages, organs [1], environment [2], artificial tending measures and even different lineages [3]. The information provided from transcriptome studies of different experimental conditions or tissues can help in the characterization of important traits related to secondary metabolite formation and to test the molecular mechanisms associated to these metabolites [4-6]. Euscaphis is a valuable ornamental and medicinal plant from the Staphyleaceae Family, which contains two species in China-Euscaphis japonica Dippel and Euscaphis konishiii Hayata. It has been widely used to treat headaches, dizziness, colds, urticaria, hernia, and rheumatism according to Flora of $\mathrm{Fu}-$ jian Province. Until now, several kinds of compounds have been isolated from Euscaphis, such as triterpene compounds [7-10], phenolic acid compounds [11, 12], flavonoid compounds [9, 11] and others [11, 13, 14]. Anti-cancer, anti-inflammatory and antifibrotic activities have been demonstrated from Euscaphis extracts from different organs by modern pharmacological research, showing that the major active ingredients were triterpenes, flavonoids and phenolic acid [7-9, 11]. Several kinds of flavonoid compounds have been isolated from E. konishii capsule in our previous study (in press). However, the molecular mechanism of active ingredients biosynthesis and accumulation was still unclear because of the lack of E. konishiii genomic data.

Flavonoids are the most common and widely distributed polyphenolic secondary metabolites in plants. Flavonoids have many biochemical properties, such as antioxidant $[15,16]$, anticancer $[17,18]$, hepatoprotective $[19,20]$, antiviral $[21,22]$, anti-inflammatory $[23,24]$ and antibacterial activity $[17,25]$. In response to the abundant biochemical activities, research groups have directed their attention to the flavonoid biosynthesis and accumulation mechanism in plants. Until now, the biosynthesis pathway of flavonoids has been well studied in some plants such as Vaccinium macrocarpon Ait. [26] and Dracaena cambodiana [27]. Given the variable distribution and contents of those flavonoids among different plants, organs, origins and even lineages, the molecular mechanisms of flavonoid biosynthesis, transport and regulation might be diverse and complex. Consequently, it is necessary to study the molecular mechanism of flavonoids biosynthesis and regulation in E. konishii. However, the lack of genomic and transcriptomic data makes difficult to study these mechanisms in E. konishii.

RNA sequencing (RNA-seq), which uses next-generation sequencing (NGS) to reveal the presence and quantity of RNA in a biological sample at a given moment in time [28], have been widely used as a powerful and valid tool to reveal gene expression patterns of any given condition due to high-throughput, accuracy and reproducibility [29]. In this study, high-throughput sequencing was performed to describe the transcriptome of E. konishiii in three different tissues to further understand the molecular mechanism of flavonoid biosynthesis.

\section{Results}

Rutin quantitative determination in E. konishii tissues

Rutin content in different E. konishii tissues was determined by HPLC, results are shown in Table 1 and Fig. 1 . Quercetin-3-O-rutinoside (rutin) quantification shows that there is significant difference among different tissues. Leaf showed the highest content of rutin with $26.31 \pm 2.43 \mathrm{mg} \cdot \mathrm{g}^{-1}$, while the branch showed the lowest rutin amount $\left(1.43 \pm 0.16 \mathrm{mg} \cdot \mathrm{g}^{-1}\right)$.

\section{Transcript sequencing and assembly}

Three mRNA libraries were generated from leaf, branch and capsule of E. konishii using Illumia sequencing technology. Adaptor sequences and low-quality reads were removed, approximately 91.7, 100.3 and 100.1 million clean reads were acquired; moreover, 27.11, 29.61 and 29.63 gigabase pairs of nucleotides were generated and the GC percentages were 45.13, 45.62 and 45.10 for leaf, branch and capsule, respectively (Table 2). Finally, a total of 85,342 unigenes with a mean length of $893.60 \mathrm{bp}$ and N50 length of $1307 \mathrm{nt}$ were assembled by Trinity program from the three tissues. The length distribution of all unigenes are shown in Fig. 2. The raw data has been submitted to NCBI, Sequence Read Archive (SRA) submission: SUB4637004.

\section{Function annotation}

The assembled unigenes were searched against NCBI non-redundant (Nr), Swiss-Prot, KEGG (Kyoto Encyclopedia of Genes and Genomes) pathway, GO (Gene Ontology) and COG (Clusters of Orthologous Groups of proteins) databases using BLAST $\left(\mathrm{e}<10^{-5}\right)$. A total of $40,218(47.13 \%$ of 85,342$)$ unigenes were annotated to one or more functions from these databases, 39,074 (45.79\%), 23,899 (28\%), 11,713 (13.72\%), 24,234 (28.40\%), 14,291(16.75\%) from $\mathrm{Nr}$ database, GO, COG, Swiss-Prot and KEGG databases, respectively (Table 2).

A total of 23,899 (28\%) unigenes were annotated into GO terms (Fig. 3). The unigenes were classified into 54

Table 1 Quercetin-3-O-rutinoside (rutin) contents in three $E$. konishii tissues

\begin{tabular}{llll}
\hline & Leaf & Branch & Capsule \\
\hline Rutin content $/ \mathrm{mg} \cdot \mathrm{g}^{-1}$ & $26.31 \pm 2.43 \mathrm{a}$ & $1.43 \pm 0.16 \mathrm{c}$ & $7.29 \pm 0.89 \mathrm{~b}$ \\
\hline
\end{tabular}

Data are represented as mean \pm SD, different words indicate significant difference of the rutin content based on three biological replications. $(P<0.05$, $\mathrm{t}$-test; $\mathrm{n}=3$ ) 


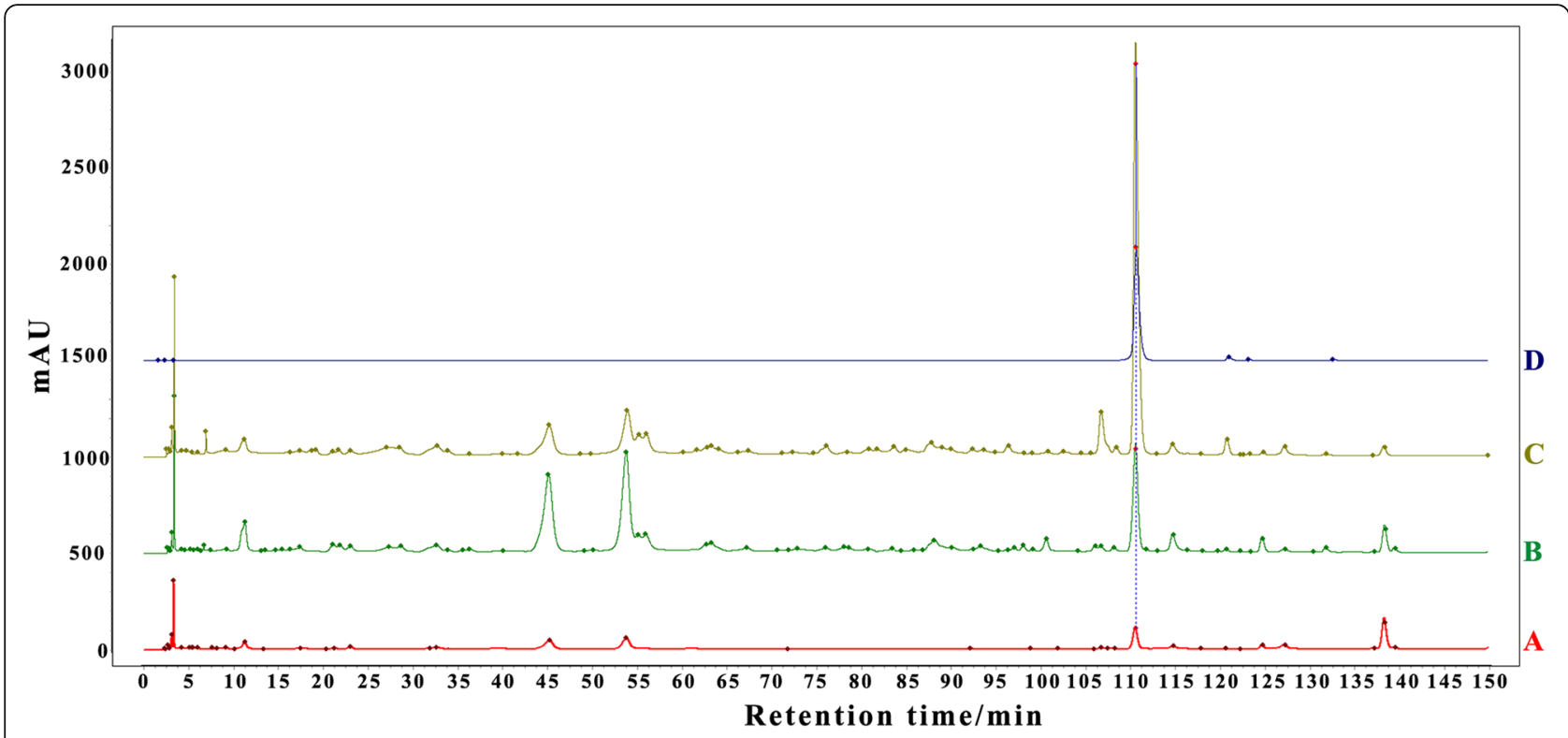

Fig. 1 Typical chromatograms for determining rutin in three organs of E. konishii. (A) Branch; (B) Capsule; (C) Leaf; (D) Rtuin

subcategories within three standard categories (molecular functions, biological processes and cellular components). "Cell" and "cell part" were the top terms in the cellular process domain, in molecular function category, "catalytic activity" and "bingding" were the highest enriched, while "metabolic process" and "cellular process" were the most enriched in the biological process domain.

COGs analysis results showed a total of 11,713 (13.72\%) unigenes annotated to COG functional classes. "General function prediction only" was the largest group, followed by "replication, recombination and repair", "transcription", "translation, ribosomal structure and biogenesis", "posttranslational modification, protein turnover, chaperones", "signal transduction mechanisms", "carbohydrate transport and metabolism" and "energy production and conversion" (Fig. 4).

KEGG (Kyoto Encyclopedia of Genes and Genomes) pathway analysis in this study was performed to identify biochemical pathway active in the branch, capsule and leaf of E. konishii. A total of 14,291 (16.75\%) unigenes were assigned to 128 KEGG pathways (Additional file 1), covering five major KEGG categories. In the biosynthesis of secondary metabolites category, 900 unigenes were annotated into 22 KEGG secondary metabolites, "phenylpropanoid biosynthesis pathway" was the largest group, followed by "terpenoid backbone biosynthesis", "steroid

Table 2 Summary of the assembly and annotation of the transcriptome

\begin{tabular}{lclr}
\hline & Leaf & \multicolumn{1}{l}{ Branch } & \multicolumn{1}{c}{ Capsule } \\
\hline Clean reads & $91,768,881$ & $100,124,694$ \\
Clean nucleotides(nt) & $27,117,135,514$ & $29,363,012$ & $29,636,461,864$ \\
GC percentage (\%) & 45.13 & 45.62 & 45.10 \\
Combined non-redundant unigene & & 85,342 & $76,261,599$ \\
Total length & 893.60 \\
Mean length & 1307 \\
N50(nt) & $39,074(45.79 \%)$ \\
Nr & $23,899(28 \%)$ \\
GO & $11,713(13.72 \%)$ \\
COG & $24,234(28.40 \%)$ \\
Swiss-Prot & $14,291(16.75 \%)$ \\
KEGG & $40,218(47.13 \%)$ \\
All annotated & &
\end{tabular}




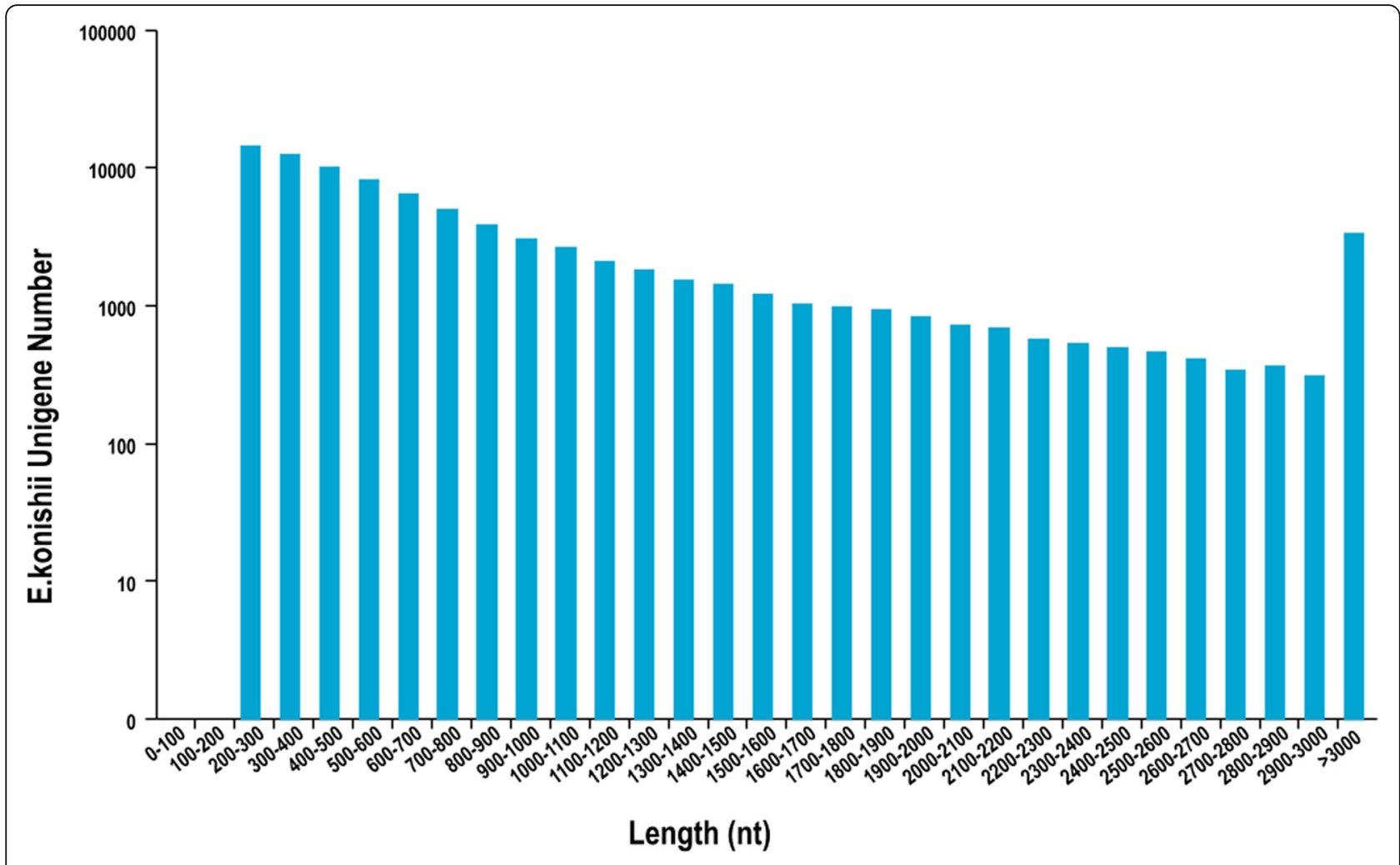

Fig. 2 Unigenes length distribution.The y-axis number has been converted into logarithmic scale

biosynthesis", "carotenoid biosynthesis", "tropane, piperidine and pyridine alkaloid biosynthesis", "arachidonic acid metabolism", "linoleic acid metabolism" and "flavonoid biosynthesis".

\section{Differential gene expression among tissues}

Differential expressed genes between two groups were identified by DESeq. A total of 4871 genes were differentially expressed between leaf and branch (2878 up-regulated and 1993 down-regulated in branch compared with leaf), the number of DEG between leaf $v s$ capsule and branch vs capsule was 3474 (1814 up-regulated and 1660 down-regulated) and 2910 (1086 up-regulated and 1824 down-regulated), respectively (Table 3, Fig. 5).

\section{GO enrichment and KEGG pathway analysis of DEGs}

To further identify the biological functions of the DEGs, the DEGs were blast against GO and KEGG databases. A total of 2515, 1786 and 1571 DEGs were annotated from leaf $v s$ branch, leaf $v s$ capsule, and branch $v s$ capsule, respectively. "Catalytic activity", "cellular process", "single-organism process", "organic substance metabolic process, binding and primary metabolic process" were the most enriched GO terms in leaf-branch DEGs; while in leaf-capsule DEGs, "metabolic process", "cellular process", "single-organism process", “organic substance metabolic process", "primary metabolic process" and "cell part" were the most annotated terms; and in branch-capsule the "metabolic process", "catalytic activity", "single-organism process", "cellular process", "binding and organic substance metabolic process" were the most abundant terms (Additional file 2).

In leaf-branch DEGs, 1247 unigenes were mapped to 109 KEGG pathways, and the most significantly enriched metabolic were "phenylpropanoid biosynthesis", "cyanoamino acid metabolism", "carotenoid biosynthesis", "starch and sucrose metabolism", "plant hormone signal transduction, photosynthesis" and "flavonoid biosynthesis". In contrast, leaf-capsule DEGs, comprised 877 unigenes that were assigned into 108 KEGG pathways: "photosynthesis, phenylalanine metabolism", "phenylpropanoid biosynthesis", "cyanoamino acid metabolism", "flavonoid biosynthesis", "photosynthesis- antenna proteins", "carotenoid biosynthesis", "pentose and glucuronate interconversions", "diterpenoid biosynthesis", "glycine, serine and threonine metabolism" were the most significantly enriched pathways. Finally, branch-capsule DEGs, comprised 777 unigenes that were represented in 107 KEGG pathways, and "plant hormone signal transduction", "diterpenoid biosynthesis", "phenylalanine metabolism", "starch and sucrose metabolism", "taurine and hypotaurine metabolism", "carotenoid biosynthesis", "cyanoamino acid metabolism", "isoflavonoid biosynthesis", "phenylpropanoid biosynthesis", "galactose 


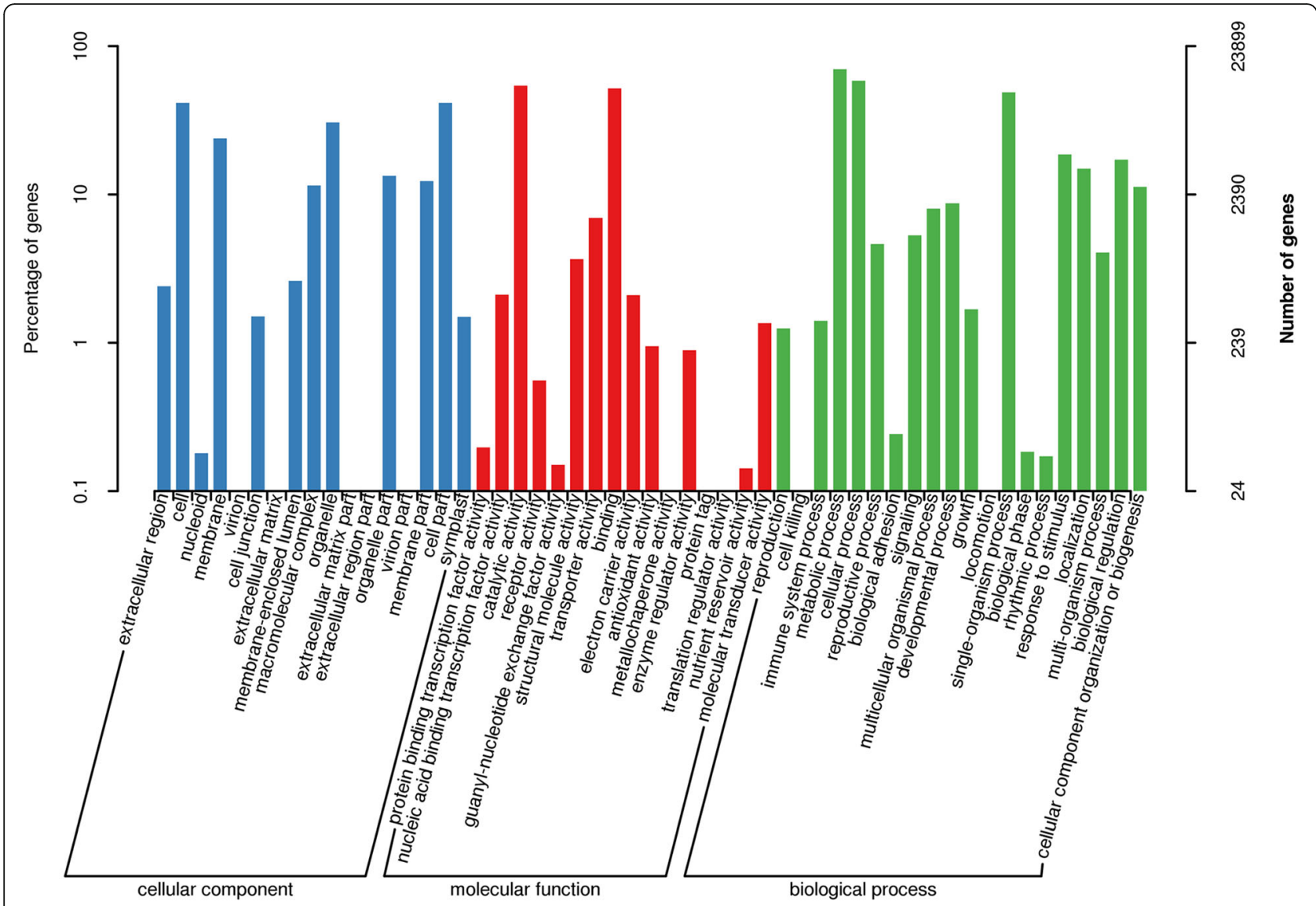

Fig. 3 Gene ontology classification of E. konishiii transcriptome. Unigenes were annotated in three categories: cellular components, molecular functions, and biological process. Right $y$-axis indicates the number of genes in a category; left $y$-axis indicates the genes percentage in a specific

metabolism" and "pentose and glucuronate interconversions" were the most significantly enriched pathways (Additional file 3).

\section{Candidate genes involved in flavonoid biosynthesis}

Given that E. konishiii is rich in flavonoids, candidate genes involved in flavonoids biosynthesis were identified in this study. In plants, the flavonoids biosynthesis pathway has been studied in several species, such as the model plant Arabidopsis thaliana [30], important crops like Vitis vinifera [31, 32], Zea mays [33] and Hordeum vulgare [34]. However, the mechanism of flavonoids biosynthesis in E. konishiii is still not understood.

We identified the genes encoding the enzymes involved in flavonoid biosynthesis pathway in the annotated $E$. konishiii transcriptome. A brief schematic of flavonoids biosynthesis pathway is shown in Fig. 6a, which was modified from KEGG databases. Flavonoids are synthesized in the cytosol from Coumaroyl-CoA, which is synthesized from phenylalanine by the enzymes phenylalanine ammonia-lyase (PAL, 12 unigenes), cinnamate 4-hydroxylase $(\mathrm{C} 4 \mathrm{H}, 4$ unigenes) and 4 coumarate CoA ligase (4CL,16 unigenes). Coumaroyl-CoA can be converted either to naringenin under the action of chalcone synthase (CHS, 11 unigenes) and chalcone isomerase (CHI, 1 unigene) or to eriodictyol by the enzymes shikimate O-hydroxycinnamoyltransferase (HCT, 8 unigenes), coumaroylquinate3'-monooxygenase (C3'H, 3 unigenes), caffeoyl-CoA O-methyltransferase (CCoAMT, 6 unigenes) and chalcone synthase (CHS, 11 unigenes); flavanone 3-hydroxylase (F3H, 2 unigenes) converts the naringenin to dihydrokaempferols (DHK) and then are catalyzed to dihydroquercetins (DHQ) by flavonoid 3'-hydroxylase (F3' $\mathrm{H}, 1$ unigene) or dihydromyricetins (DHM) by flavonoid 3', 5' -hydroxylase (F3'5'H, 3 unigenes). Finally, DHK, DHM and DHQ are converted to flavonols by flavonolsynthese (FLS, 2 unigenes). In the anthocyanin branch, dihydroflavonol 4-reductase (DFR, 2 unigenes) catalyzes DHQ and DHM to leucocyanidins and leucodelphinidins and then continually converted to cyanidin or delphindin by leucoanthocyanidin dioxygenase (ANS, 1 unigene). The modification reactions, such as glycosylation, hydroxylation and methylation by enzymes UDP-glycosyltransferase (UGT) [35], Cytochrome P450 (CYP) and O-methyltransferase (OMT) generate a variety of flavonoids. In this study, 40 UGT, 122 CYP and 25 OMT unigenes were found. 


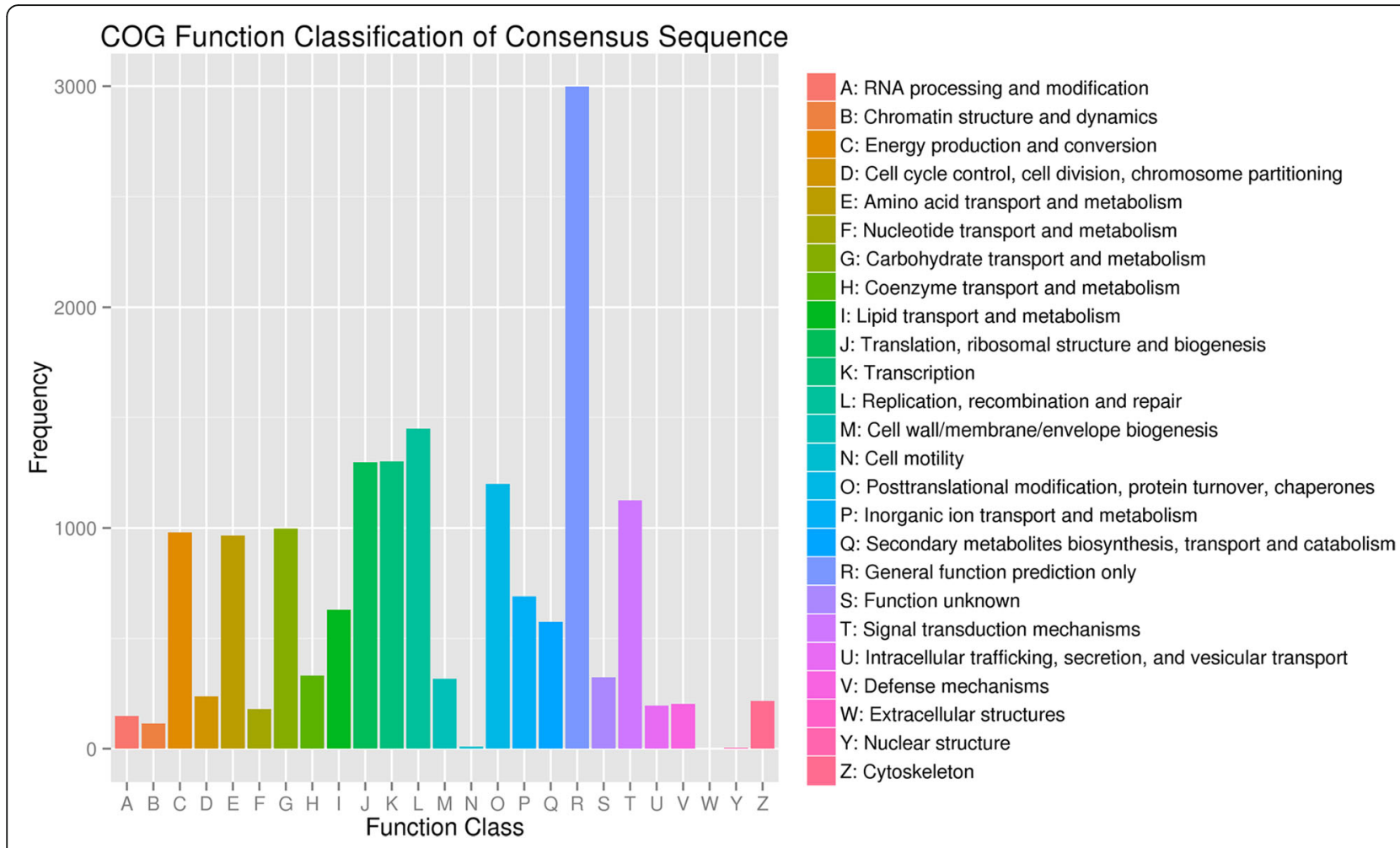

Fig. 4 COG function classification of E. konishiii unigenes. All putative unigenes were analyzed using the COG database. COG classifications were divided into 26 functional categories; 11,713 classified unigenes were assigned to 25 COG classifications

Furthermore, the expression patterns of all the transcripts encoding enzymes involved in the flavonoids biosynthesis steps were analyzed. $2 \mathrm{PAL}, 2 \mathrm{C} 4 \mathrm{H}, 3 \mathrm{CLL}, 2$ HCT, 1 C3'H, 2 CHS, 1 CHI, 1 F3H, 1 F3'5'H, 1 LAR, 1 ANS, $20 \mathrm{CYP}$ and 5 OMT unigenes were up-regulated and 1 CHS, 1 FLS, 8 UGT, 25 CYP and 1 OMT unigenes were down regulated in branch against in leaf. In capsule against leaf, 2 PAL, 3 4CL, 1 HCT, 1 C3' $\mathrm{H}, 2$ CHS, 1 CHI, 1 F3H, 1 F3'5'H, 1 LAR,1 ANS, 7 UGT, 16 CYP and 5 OMT unigenes were up-regulated and $1 \mathrm{HCT}, 1$ FLS, 2 UGT, 9 CYP and 2 OMT unigenes were down

Table 3 Statistical table of differently expressed genes (DEGs) and annotation

\begin{tabular}{llll}
\hline Type & Leaf vs Branch & Leaf vs Capsule & Branch vs Capsule \\
\hline number & 4871 & 3474 & 2910 \\
up-regulated & 2878 & 1814 & 1086 \\
down-regulated & 1993 & 1660 & 1824 \\
COG & 1178 & 825 & 706 \\
GO & 2525 & 1786 & 1571 \\
KEGG & 1247 & 877 & 777 \\
Swiss-port & 2833 & 1996 & 1816 \\
Nr & 3889 & 2738 & 2414 \\
All annotated & 3928 & 2755 & 2429 \\
\hline
\end{tabular}

regulated. In capsule against branch, 1 CHS, 2 CCoAMT, 11 UGT, 21 CYP and 2 OMT unigenes were up-regulated and 2 C4H, 2 HCT, 1 CHS, 1 F3'5'H, 2 UGT, 13 CYP and 2 OMT unigenes were down-regulated (Fig. 6b, Additional file 4). Differential gene expression among different tissues might play important roles in flavonoid biosynthesis in E. konishiii.

\section{Candidate genes involved in flavonoid transport}

In plants, flavonoids are synthesized in the cytosol and then stored into vacuoles for storage or to be transported to other locations [36]. According to Zhao et al. (2010), there are three major mechanisms proposed for flavonoid transport: membrane vesicle-mediated transport (MVT), membrane transporter-mediated transport (MMT) and glutathione-S-transferases (GST) [37]. Several genes responsible for these mechanisms have been identified in Arabidopsis [38, 39], M. truncatula [40], Zea mays [41] and $V$. vinifera [42, 43].

ATP-binding cassette transporters (ABC) (G-type (APCG) and the multidrug resistance-associated protein (MRP)-type), $\mathrm{H}^{+}$-ATPases, multidrug and toxic compound extrusion protein (MATE) transporters and $\mathrm{H}^{+}$-Ppase belong to MMT, while solube N-ethylmaleimidesensitive factor attachment protein receptors (SNARE) and vacuolar sorting receptor (VSR) are responsible for MVT. In $E$. 

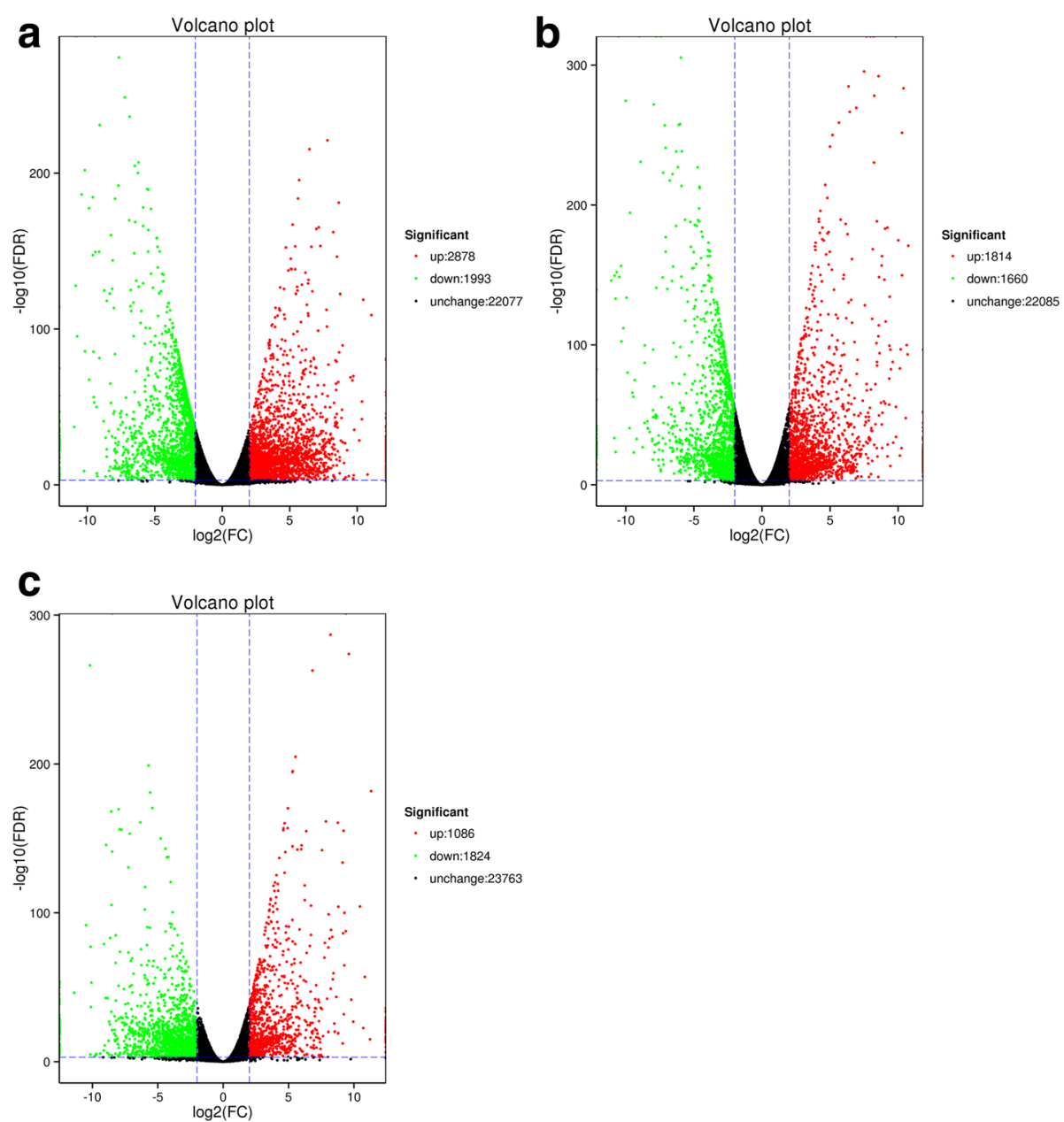

Fig. 5 Gene differential expression in E. konishiii transcriptome. Volcano plot shows the differential expressed genes. (a) Leaf vs Branch, (b) Leaf vs Capsule, (c) Branch vs Capsule. (black spot: unchanged unigenes, green spot: down-regulated unigenes and red spot: represent up-regulated unigenes)

konishiii, we found 29 unigenes encoding MATE, 175unigenes encoding MRP/ABCG, 19 unigenes encoding $\mathrm{H}^{+}$-ATPase, 56 unigenes encoding GST, 14 unigenes encoding SNARE and 2 unigenes encoding VSR. Expression patterns of all the above described genes were analyzed, moreover, $24 \mathrm{ABC} / \mathrm{MRP}, 2 \mathrm{GST}, 1 \mathrm{H}+$-ATPase and 2 MATE unigenes were up-regulated and 7ABC/MRP, 4 GST, $1 \mathrm{H}+$-ATPase, 2 MATE unigenes were down-regulated in branch $v s$ leaf. In capsule $v s$ leaf, 13 ABC/MRP, 6 GST, $1 \mathrm{H}+$-ATPase and 2 MATE unigenes were up-regulated and 7 ABC/MRP, 2 GST, and 2 MATE unigenes were down-regulated. Finally, 9 ABC/MRP, 6 GST and 2 MATE unigenes were up-regulated and $10 \mathrm{ABC} /$ MRP, 2 MATE unigenes were down-regulated in capsule $v s$ branch (Additional file 4).

\section{Candidate transcription factors involved in flavonoid biosynthesis and transport}

Recently, it has been showed that structural genes involved in flavonoids are controlled by MYB protein family, basic helix-loop-helix (bHLH) transcription factors (TFs) and WD-repeat-containing proteins [44, 45]. MYB TFs are one of the largest TF families and plays important roles in controlling cellular processes, such as development, responses to biotic and abiotic stresses [46], differentiation [47] and metabolism [48, 49]. The bHLH, a superfamily of transcriptome factors (TFs), has been demonstrated to display different biological functions in development of plants [50]. WD-repeat-containing proteins (WDR) are implicated in a variety of functions ranging from signal transduction and transcription regulation to cell cycle control, autophagy and apoptosis [51]. .

We identified 97 MYB, 84 bHLH and 39 WD40 unigenes. Transcript analysis showed that $20 \mathrm{MYB}, 12 \mathrm{bHLH}$ and 1 WD40 unigenes were up-regulated and 3MYB, 2 bHLH and 3 WD40 unigenes were down-regulated in branch $v s$ leaf. Moreover, 10 MYB, 5 b HLH unigenes were up-regulated and 4 MYB, 2 bHLH and 3 WD40 unigenes were down-regulated in capsule $v s$ leaf. Finally, 8 MYB, 4 bHLH and 1 WD40 unigenes were up-regulated 

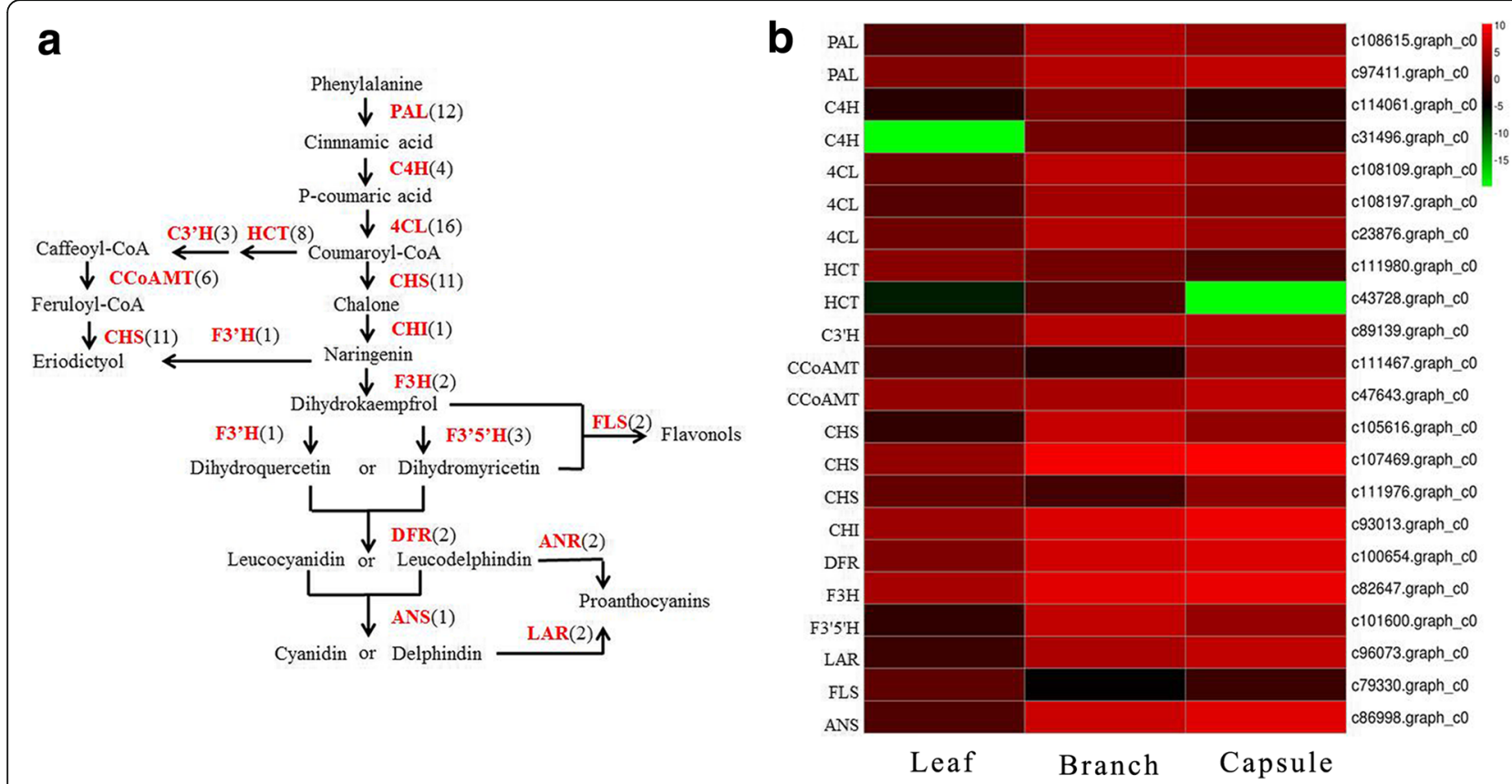

Fig. 6 Putative flavonoids biosynthesis pathway in E. konishiii. (a) Pathway for flavonoids biosynthesis. The numbers in brackets following each gene name indicate the number of E. konishiii unigenes corresponding to that gene. Enzyme abbreviations are as follows: PAL, phenylalanine ammonia lyase; $\mathrm{C} 4 \mathrm{H}$, cinnamate 4-hydroxylase; $4 \mathrm{CL}$, 4-coumarate CoA ligase; $\mathrm{HCT}$, shikimate O-hydroxycinnamoyltransferase; $C 3^{\prime} \mathrm{H}$, coumaroylquinate 3'-monooxygenase; CCOAMT, caffeoyl-CoA O-methyltransferase; CHS, chalcone synthase; $\mathrm{CHI}$, chalcone isomerase; $\mathrm{F3H}$, flavanone 3-hydroxylase; F3'H, flavonoid 3'-hydroxylase; F3'5'H, flavonoid 3',5'-hydroxylase; FLS, flavonolsynthese; DFR, dihydroflavonol 4-reductase; ANR, anthocyanidinreductase; ANS, leucoanthocyanidin dioxygenase; LAR, leucoanthocyanidin reductase. (b) Expression levels of candidate $E$. konishiii unigenes coding for key enzymes involved in flavonoids biosynthesis pathways. Green and red colors are used to represent low-to-high expression levels, and color scales correspond to the mean centered $\log _{2}$-transformed FPKM values

and 15 MYB, 12 bHLH unigenes were down-regulated in capsule $v s$ branch (Additional file 4). These different expression profile of transcription factors might be responsible for regulating flavonoid biosynthesis and transport in E. konishii .

\section{Phylogenetic tree analysis of key genes involved in flavonoid biosynthesis}

Phylogenetic tree analysis of key genes involved in flavonoid biosynthesis was carried using MEGA and the neighbor joining method with 1000 bootstrap replicates, and the results were shown in Additional file 5. The results demonstrated that all the genes involved in flavonoid biosynthesis are homologous gene of one or more known genes.

\section{Quantitative real-time PCR (qRT-PCR) validationof DEGs involved in flavonoid biosynthesis}

To further analyze the consistency of RNA-seq data in this study, a total of 15 genes involved in flavonoid biosynthesis were selected for qRT-PCR validation. The details of those unigenes and primer pairs used in this study are shown in Additional file 6. A comparative analysis of all the selected genes showed a similar expression pattern in
qRT-PCR analysis as observed in RNAseq data (Fig. 7), suggesting the reliability in results.

\section{Discussion}

Rutin accumulation in different organs of $E$. konishii

We isolated a many kinds flavonoids in the previous studies, and the rutin is the most abundant flavonoid in E. konishii, which has a long history of use in nutritional supplements for its action against oxidative stress, inflammation, and hyperglycemia [52]. HPLC method was carried in this study to mesure rutin content in different organs (Leaf, Branch and Capsule) of E. konishii. Distinctively, the leaf had significantly higher content of rutin compared with other organs, which might signify the essential role of rutin in plant development and physiology.

\section{Illumina sequencing and sequence annotation}

E. konishii is a very important traditonal Chinese mdicine. Even though flavoniods are one of the most important active constituents of E. konishii, little is known about the mechanisms responsible for flavonoid biosynthesis and metabolism. The aim of this study were to generate a large amount of cDNA sequence data that would facilitate more detailed studies in E. konishii, and 


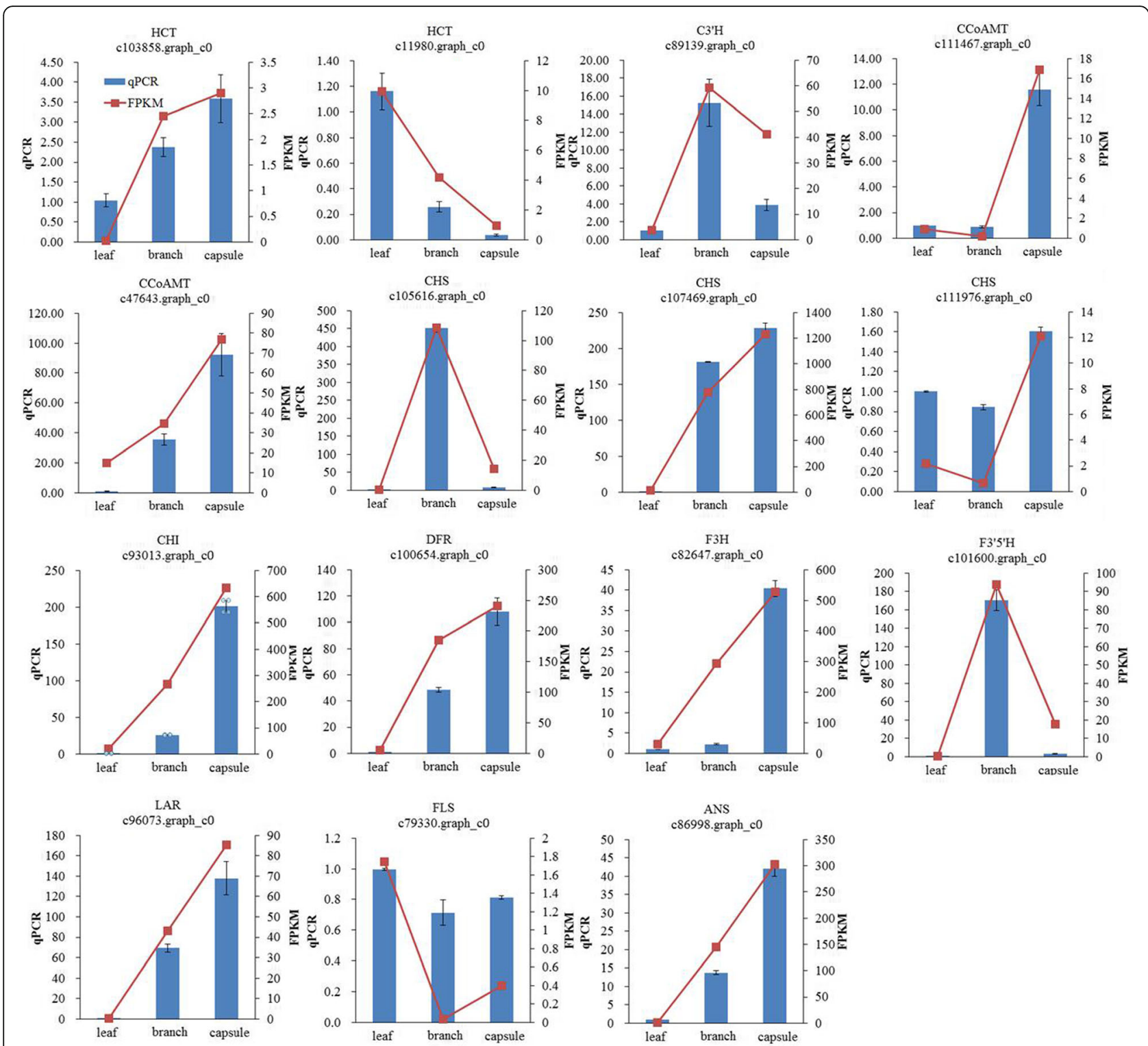

Fig. 7 RNA-seq data validation by qRT-PCR. The histograms show the qPCR results of 15 unigenes involved in flavonoid biosynthesis among tissues of E. konishii, the red line charts show the FPKM values of these unigenes, and blue bars show the qPCR results, represent the mean \pm SD of three biological replicates. The left Y-axis indicates the relative expression levels calculated by qPCR and the right Y-axis indicates the FPKM values of RNA-seq data

to identify the genes related to flavonoid biosynthesis and accumulation. In this study, a total of 85,342 unigenes were generated by Illumna sequencing from leaf, branch and capsule of E. konishii, and 40,218 (47.13\% of 85,342 ) unigenes provided a significant Blast result. This imformation can provide as equate resources to sthudy E. konishii and other related species. But, The annotation percentage of unigenes assembled in this study is lower than other studies, such as Erigeron breviscapus (61.7\%) [53] and Hypericum perforatum (68.86\%) [54], this may be due to the length and integrity of the sequences obtained by transcriptome splicing or the lack of genomic information for E. konishii and its related species, and it needed to be further study in the future.

\section{DEGs may play crucial roles in organ function and morphogenesis in E. konishii}

Higher plants comprise several organs made up of various tissues and cell types. Gene expression patterns differed among the organs of E. konishii in our study. Both DEGS were subjected to GO and KEGG annotation, the results promoted our understanding of the genes expression patterns among organs. Most DEGs annotated in basic functions such as "metabolic process", "cellular 
process", "single-organism process", "organic substance metabolic process", "primary metabolic process". But, the DEGs assigned into different KEGG pateways due to the organ-specific manner, such as in leaf, DEGs were significantly enriched in pathways of "photosynthesis, phenylalanine metabolism", "photosynthesis- antenna proteins" and "carotenoid biosynthesis". and in branch, DEGs were higher enriched function of "plant hormone signal transduction", "diterpenoid biosynthesis" and "phenylalanine metabolism". A functional analysis using the GO and KEGG classification system of DEGs can provide a new insight for studying orgam-specific processes, functions and pathways among different $E$. konishii tissues.

\section{Candidate genes involved in flavonoid biosynthesis}

The relative expression level of candidate genes involved in flavonoids biosynthesis was studied by qRT-PCR, and the results were shown in Fig. 7. As the results showed, the candidate genes involved in flavonoids represent different expression patterns in different organs of E. konishii. CHS (c107469.graph_c0), CHI (c93013.graph_c0), DFR (c100654.graph_c0), F3H (c82647.graph_c0), LAR (c96073.graph_c0) and ANS (c86998.graph_c0) showed the same expression pattern in different organs, were all up-regulated in capsule than leaf and branch, and our previous study showed that there were a large amount of anthocyanins in the capsule of E. konishii, and the total content of anthocyanins reached $3.880 \mathrm{mg} \cdot \mathrm{g}^{-1}$ [55]. It suggests that the above-mentioned up-regulated genes in capsule may play important roles in the biosynthesis of anthocyanins in capsule of E. konishii. We measured the content of rutin in three organs of E. konishii by HPLC method, it was significant difference among organs, and in leaf was highest, reached $26.31 \pm 2.43 \mathrm{mg} \cdot \mathrm{g}^{-1}$, in branch was lowest $\left(1.43 \pm 0.16 \mathrm{mg} \cdot \mathrm{g}^{-1}\right)$, and also the expression level of FLS (c79330.graph_c0), a key gene involved in flavonols biosynthesis, was up-regulated in leaf compared with branch and capsule, and up-regulated in capsule compared with branch, this is consistent with the content of rutin, a flavonol glycoside. These findings suggest that the FLS (c79330.graph_c0) gene may be responsible for the organ-specific biosynthesis of rutin in E. konishii.

It has been reported that MYB-bHLH-WD40 complexes can regulate flavonoid biosynthesis pathway through an intricate network [56, 57]. An BoMYB2 together with various BobHLHs from Brassica oleracea L. var. botrytis specifically regulated the late anthocyanin biosynthetic pathway genes for anthocyanin biosynthesis [58]. An R2R3-MYB transcription factor, TaMYB14 from Trifolium arvense activate proanthocyanidin biosynthesis [59]. An R2R3-MYB transcription factor VvMYBF1 isolated from
Vitis vinifera 'Shiraz' to be a specific activator of flavonol synthase1 (FLS1). We identified 97 MYB unigenes, and 7 MYB (Additional file 4) unigenes were up-regulated in capsule compared with leaf and branch, which suggested that this MYB genes may be responsible for anthocyanin biosynthesis in capsule of E. konishii, and another MYB gene (c47321_c0) showed the consistently expression pattern with FLS gene (c79330.graph_c0), it may play important role in regulate the organ-specific biosynthesis of rutin of E. konishii.

\section{Conclusion}

In this study, the comparative transcriptome analysis of leaf, branch and capsule of E. konishii was performed. We identified putative transcripts involved in biosynthesis and accumulation of flavonoids, and their different expression pattern in the leaf, branch and capsule tissues of E. konishii was calculated by DESeq method, which suggests tissues-specific biosynthesis, accumulation and modification of flavonoids might occur in different tissues. This study will contribute significantly to further molecular research of E. konishii and other related species.

\section{Materials and methods}

\section{Plant materials}

One year old leaf, branch and capsule of E. konishii (Fig. 8) were collected from Fujian Agriculture and Forestry University, Fujian Province, China during November 15th 2016. All samples were harvested, washed and surface dried and then frozen in liquid nitrogen immediately and stored at $-80^{\circ} \mathrm{C}$ until required for further processing. Three biological replicates of each organ were used for RNA extraction and transcriptome sequencing.

\section{Reagends and standards}

Methanol of HPLC grade was from Merck (Darmstadt, Germany), other reagents were of analytical grade, rutin (purity $>98 \%$ ) was isolated in our laboratory, and its purity and structure was confirmed by HPLC and by comparison of spectral data to published in the literature.

\section{Quantitative analysis of Rutin in E. konishii Instrumentation and chromatographic conditions}

HPLC-DAD was carried out by an Waters HPLC-DAD system comprising a vacuum degasser, binarypump, autosampler, thermostated column compartment, and DAD (Waters, USA), which was used for acquiring chromatograms and UV spectra. An Alltima C18 column $(5 \mu \mathrm{m} ; 4.6 \times 400 \mathrm{~mm})$ was used for HPLC analysis. The mobile phase consisted of $0.1 \%$ acetic acid in water (A) and methanol (B), and the procedure was performed with a gradient program of $10-16 \%$ (B) at $0-30 \mathrm{~min}$, $16-16 \%$ (B) at $30-40 \mathrm{~min}, 16-30 \%$ (B) at $40-80 \mathrm{~min}$, and $30-60 \%$ (B) at $80-150 \mathrm{~min}$. The flow rate was $1 \mathrm{~mL} / \mathrm{min}$. 


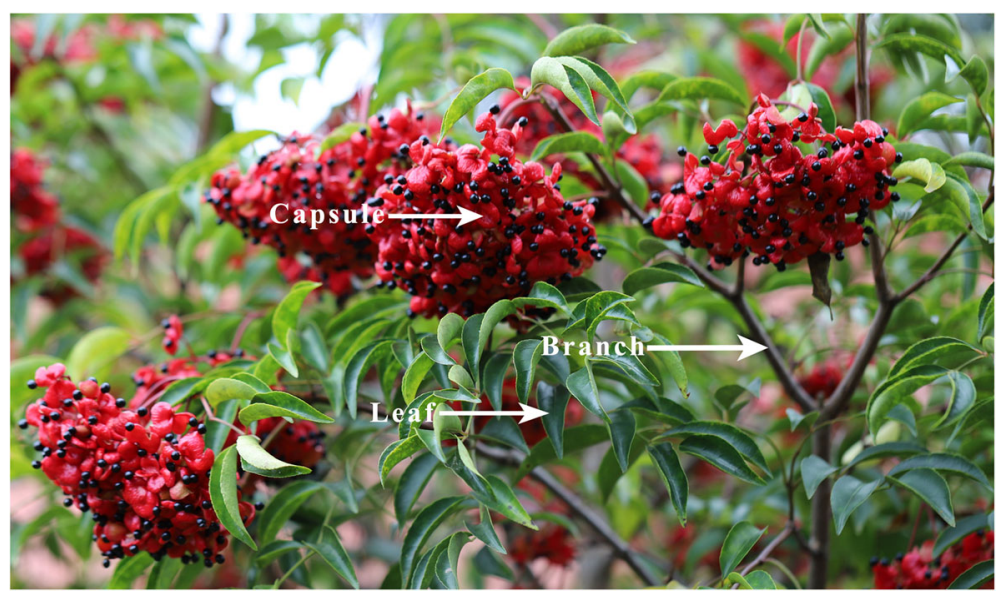

Fig. 8 The three organs of E. konishii used in this study. This picture is captured by the first author of this manuscript

The detection wavelength was set at $254 \mathrm{~nm}$. The column temperature was set at $30^{\circ} \mathrm{C}$. The injection volume was $10.0 \mu \mathrm{L}$.

\section{Preparation of standard and sample solutions}

Standard solutions were prepared at a concentration of $1 \mathrm{mg} / \mathrm{mL}$ with HPLC grade methanol. About $5.0 \mathrm{~g}$ of each dried sample was ground into powder and extracted with $10 \mathrm{~mL}$ of $70 \%$ methanol at $80^{\circ} \mathrm{C}$, for two times, each for an hour. The solvent was condensed in vacuum to yield a crude extract, and the crude extract was dissolved by methanol and made up to $50 \mathrm{~mL}$.

\section{RNA extraction and library preparation for transcriptome sequencing}

Total RNA was extracted using the Tiangen reagent kit (DP441). A total amount of $3 \mu \mathrm{g}$ RNA per sample was used as input material for the RNA sample preparations. Sequencing libraries were generated using NEBNext ${ }^{\circ} \mathrm{Ul}-$ tra $^{\text {Tu }}$ RNA Library Prep Kit for Illumina ${ }^{\circ}$ (NEB, USA) following manufacturer's protocol. Briefly, mRNA was purified from total RNA using poly-T oligo-attached magnetic beads. Fragmentation was carried out using divalent cations under elevated temperature in NEBNext First Strand Synthesis Reaction Buffer (5X). First strand cDNA was synthesized using random hexamer primer and M-MuLV Reverse Transcriptase (RNase H-). Second strand cDNA synthesis was subsequently performed using DNA Polymerase I and RNase H. Remaining overhangs were converted into blunt ends via exonuclease/ polymerase activities. After adenylation of 3' ends, NEBNext Adaptor with hairpin loop structure were ligated to prepare for hybridization. In order to select cDNA fragments of preferentially $150 \sim 200 \mathrm{bp}$ in length, the library fragments were purified with AMPure XP system (Beckman Coulter, Beverly, USA). $3 \mu \mathrm{L}$ USER Enzyme (NEB, USA) was used with size-selected, adaptor-ligated cDNA at $37^{\circ} \mathrm{C}$ for $15 \mathrm{~min}$ followed by $5 \mathrm{~min}$ at $95^{\circ} \mathrm{C}$ before PCR. PCR was performed with Phusion High-Fidelity DNA polymerase, Universal PCR primers and Index (X) Primer. At last, PCR products were purified (AMPure $\mathrm{XP}$ system) and library quality was assessed on the Agilent Bioanalyzer 2100 system.

\section{Clustering and sequencing}

The clustering of the index-coded samples was performed on a cBot Cluster Generation System using TruSeq PE Cluster Kit v3-cBot-HS (Illumia) according to the manufacturer's instructions. After cluster generation, the library preparations were sequenced on an Illumina Hiseq 2000 platform and paired-end reads were generated.

\section{Quality control}

Raw data (raw reads) of fastq format were firstly processed through in-house perl scripts. In this step, clean data (clean reads) were obtained by removing reads containing adapter, reads containing ploy- $\mathrm{N}$ and low quality reads from raw data. At the same time, Q20, Q30, GC-content and sequence duplication level of the clean data were calculated. All the downstream analyses were based on clean data with high quality.

\section{Transcriptome assembly}

The left files (read1 files) from all libraries were pooled into one big left.fq file, and right files (read2 files) into one big right.fq file. Transcriptome assembly was accomplished based on the left.fq and right.fq using Trinity [60] with min_kmer_cov set to 2 by default and all other parameters set default.

\section{Gene functional annotation}

Gene function was annotated based on the following databases: NR (NCBI non-redundant protein sequences), KOG/COG/eggNOG (Clusters of Orthologous Groups 
of proteins), Swiss-Prot (A manually annotated and reviewed protein sequence database), KEGG (Kyoto Encyclopedia of Genes and Genomes), and GO (Gene Ontology).

\section{Quantification of gene expression levels}

Gene expression levels were estimated by RSEM [61] for each sample: 1) clean data were mapped back onto the assembled transcriptome and 2) read-counts for each gene was obtained from the mapping results.

\section{Differential expression analysis}

Differential expression analysis of two conditions/groups was performed using the DESeq R package. DESeq provide statistical procedures to determine differential expression in digital gene expression data using a model based on the negative binomial distribution. The resulting $P$ values were adjusted using the Benjamini and Hochberg's approach for controlling the false discovery rate (FDR). Genes with an adjusted $P$-value $<0.05$ found by DESeq were assigned as differentially expressed.

\section{GO enrichment analysis}

Gene Ontology (GO) enrichment analysis of the differentially expressed genes (DEGs) was implemented using topGO R packages based Kolmogorov-Smirnov test.

\section{KEGG pathway enrichment analysis}

KEGG [62] is a database resource for understanding high-level functions and utilities of the biological system, such as the cell, the organism and the ecosystem, from molecular-level information, especially large-scale molecular datasets generated by genome sequencing and other high-throughput experimental technologies (http:// www.genome.jp/kegg/). We used KOBAS software [63] to test the statistical enrichment of differential expression genes in KEGG pathways.

\section{Quantitative PCR (qRT-PCR) analysis}

Fifteen differential expressed genes (c103858.graph_c0, c111980.graph_c0, c89139.graph_c0, c111467.graph_c0, c47643.graph_c0, c105616.graph_c0, c107469.graph_c0, c111976.graph_c0, c93013.graph_c0, c100654.graph_c0, c82647.graph_c0, c101600.graph_c0, c96073.graph_c0, c79330.graph_c0, c86998.graph_c0) involved flavonoid biosynthesis were selected for quantitive real-time PCR (qRT-PCR), the primers of all selected genes were designed by Primer Premier 5 (Additional file 6). The qRT-PCR analysis of each gene was performed on a 7500 Fast ABI Real-time PCR system (Applied Bio, US) using FastStart Universial SYBR Green Master (ROCHE, Switzerland). A $20 \mathrm{uL}$ reaction mixture contain $10 \mathrm{uL}$ $2 \times$ SYBR Green Master, $0.4 \mathrm{uL}$ forward primer $(10 \mathrm{uM})$, $0.4 \mathrm{uL}$ reverse primer $(10 \mathrm{uM}), 2 \mathrm{uL}$ cDNA and $7.2 \mathrm{uLdd}$
$\mathrm{H}_{2} \mathrm{O}$ in a 96-well plates. The amplification conditions were as follows: $50^{\circ} \mathrm{C}$ for $2 \mathrm{~min}, 95^{\circ} \mathrm{C}$ for $10 \mathrm{~min}$; $40 \mathrm{cy}$ cles of $95^{\circ} \mathrm{C}$ for $15 \mathrm{~s}$ and $60^{\circ} \mathrm{C}$ for 30 s. Relative gene expression levels were calculated by $2^{-\triangle \triangle \mathrm{Ct}}, G A D P H$ and GSTU1 were selected as internal reference genes according to previous studies [64].

\section{Phylogenetic analysis}

The phylogenetic analysis based on the amino acid sequences was performed using MEGA (version 7.0, the laboratory at the Pennsylvania State University, St Collie, PA, USA) and the neighbor joining method with 1000 bootstrap replicates.

\section{Additional files}

Additional file 1: Total unigenes assigned to 128 KEGG pathways (DOCX $23 \mathrm{~kb}$ )

Additional file 2: GO annotation of DEGs (DOCX 315 kb)

Additional file 3: Significantly enriched KEGG pathways in DEGs between different tissues in E. konishii Hayata (DOCX 17 kb)

Additional file 4: Candidate genes involved in Flavonoid accumulation (DOCX $38 \mathrm{~kb}$ )

Additional file 5: Phylogenetic tree analysis of Candidate genes involved in Flavonoid biosynthesis (DOCX $15761 \mathrm{~kb}$ )

Additional file 6: Primers used in validation experiment of gene expression by qRT-PCR (DOCX $13 \mathrm{~kb})$

\section{Abbreviations}

4CL: 4-coumarate CoA ligase; ANR: Anthocyanidinreductase; ANS: Leucoanthocyanidin dioxygenase; $\mathrm{C}^{\prime} \mathrm{H}$ : Coumaroylquinate 3'monooxygenase; C4H: Cinnamate 4-hydroxylase; CCOAMT: Caffeoyl-CoA Omethyltransferase; $\mathrm{CHI}$ : Chalcone isomerase; CHS: Chalcone synthase; CYP450: Cytochrome P450.; DFR: Dihydroflavonol 4-reductase; F3'5'H: Flavonoid 3',5'-hydroxylase; F3'H: Flavonoid 3'-hydroxylase; F3H: Flavanone 3-hydroxylase; FLS: Flavonolsynthese; HCT: Shikimate Ohydroxycinnamoyltransferase; LAR: Leucoanthocyanidin reductase; OMT: Omethyltransferase; PAL: Phenylalanine ammonia lyase; UGT: UDPglycosyltransferase

\section{Acknowledgments}

Not applicable.

\section{Funding}

This work was supported by the National Science Foundation of China Projects (Grant number 31700292), the Central Financial Forestry Science and Technology Promotion Demonstration Project (grant No. Min [2017]TG01; Min [2018] TG14), and the Fujian Science and Technology Innovation Leading Talent Support Project (grant No. KRC16006A), the Peak Discipline Construction Project of Forestry of Fujian Agriculture and Forestry University (Project number 71201800709). The funding body have no role in the design of the study and collection, analysis, and interpretation of data and in writing the manuscript.

\section{Availability of data and materials}

The raw reads has been submitted to NCBI, Sequence Read Archive (SRA) accession: PRJNA431316.

Authors' contributions

WXL, LN, and SQZ conceived and designed the experiments, LJW, WHS, $X X Z$ and $X Y Y$ selected the material, WXL, LJW and WHS performed the experiments, WXL, XXZ, YLM, WH and CLR analyzed the data, WXL, CLR and SQZ wrote the paper. All authors read and approved the manuscript. 


\section{Ethics approval and consent to participate}

Not applicable.

\section{Consent for publication}

Not applicable.

\section{Competing interests}

The authors declare that they have no competing interests.

\section{Publisher's Note}

Springer Nature remains neutral with regard to jurisdictional claims in published maps and institutional affiliations.

\section{Author details}

${ }^{1}$ College of Forestry, Fujian Agriculture and Forestry University, Fuzhou, China. ${ }^{2}$ Fujian Colleges and Universities Engineering Research Institute of Conservation \& Utilization of Natural Bioresources, Fujian Agriculture and Forestry University, Fuzhou, China. ${ }^{3}$ College of Plant Protection, Fujian Agriculture and Forestry University, Fuzhou, China. ${ }^{4}$ Department of Microbiology \& Molecular Genetics, University of California, Irvine, USA. ${ }^{5}$ College of Resources and Environment, Fujian Agriculture and Forestry University, Fuzhou, China. ${ }^{6}$ College of Life Sciences, Fujian Agriculture and Forestry University, Fuzhou, China.

\section{Received: 30 January 2018 Accepted: 9 December 2018}

\section{Published online: 09 January 2019}

\section{References}

1. Park YJ, Li X, Noh SJ, Kim JK, Lim SS, Park NI, Kim S, Kim YB, Kim YO, Lee SW, et al. Transcriptome and metabolome analysis in shoot and root of Valeriana fauriei. BMC Genomics. 2016;17:303.

2. Lu N, Bernardo EL, Tippayadarapanich C, Takagaki M, Kagawa N, Yamori W. Growth and accumulation of secondary metabolites in Perilla as affected by photosynthetic photon flux density and electrical conductivity of the nutrient solution. Front Plant Sci. 2017:8:708

3. Chen M, Yang C, Sui C, Jin Y, Wei J. Zhongdanyaozhi no. 1 and Zhongdanyaozhi no. 2 are hybrid cultivars of Salvia miltiorrhiza with high yield and active compounds content. PLoS One. 2016;11(9):e0162691.

4. Hao d C, Chen SL, Osbourn A, Kontogianni VG, Liu LW, Jordan MJ. Temporal transcriptome changes induced by methyl jasmonate in Salvia sclarea. Gene. 2015;558(1):41-53.

5. Hao DC, Xiao PG. Genomics and evolution in traditional medicinal plants: road to a healthier life. Evol Bioinformatics Online. 2015;11:197-212.

6. Zhang F, Gao Q, Khan G, Luo K, Chen S. Comparative transcriptome analysis of aboveground and underground tissues of Rhodiola algida, an important ethno-medicinal herb endemic to the Qinghai-Tibetan plateau. Gene. 2014; 553(2):90-7.

7. Takahashi K, Kawaguchi S, Nishimura K, Kubota K, Tanabe Y. Studies on constituents of medicinal plants. XIII. Constituents of the pericarps of the capsules of Euscaphis japonica Pax. Chem Pharm Bull (Tokyo). 1974;22(3): 650-3.

8. Lee MK, Lee KY, Jeon HY, Sung SH, Kim YC. Antifibrotic activity of triterpenoids from the aerial parts of Euscaphis japonica on hepatic stellate cells. J Enzyme Inhib Med Chem. 2009;24(6):1276-9.

9. Cheng JJ, Zhang LJ, Cheng HL, Chiou CT, Lee IJ, Kuo YH. Cytotoxic Hexacyclic triterpene acids from Euscaphis japonica. J Nat Prod. 2010;73(10): 1655-8.

10. Li YC, Tian K, Sun LJ, Long H, Li LJ. Wu ZZ: a new hexacyclic triterpene acid from the roots of Euscaphis japonica and its inhibitory activity on triglyceride accumulation. Fitoterapia. 2016;109:261-5.

11. Lee MK, Jeon HY, Lee KY, Kim SH, Ma CJ, Sung SH, Lee HS, Park MJ, Kim YC. Inhibitory constituents of Euscaphis japonica on lipopolysaccharide-induced nitric oxide production in BV2 microglia. Planta Med. 2007;73(8):782-6.

12. Maeda H, Matsuo $Y$, Tanaka T, Kouno I. Euscaphinin, a new ellagitannin dimer from Euscaphis japonica (THUNB.) KANITZ. Chem Pharm Bull (Tokyo). 2009:57(4):421-3.

13. Takeda Y, Okada Y, Masuda T, Hirata E, Takushi A, Otsuka H. Euscapholide and its glucoside from leaves of Euscaphis Japonica. Phytochemistry. 1998; 49(8):2565-8.
14. Takeda Y, Okada Y, Masuda T, Hirata E, Shinzato T, Takushi A, Yu Q, Otsuka $\mathrm{H}$. New megastigmane and tetraketide from the leaves of Euscaphis japonica. Chem Pharm Bull (Tokyo). 2000;48(5):752-4.

15. Heim KE, Tagliaferro AR, Bobilya DJ. Flavonoid antioxidants: chemistry, metabolism and structure-activity relationships. J Nutr Biochem. 2002;13(10): 572-84.

16. Pandey AK, Mishra AK, Mishra A. Antifungal and antioxidative potential of oil and extracts derived from leaves of Indian spice plant Cinnamomum tamala. Cell Mol Biol (Noisy-le-grand). 2012;58(1):142-7.

17. Mishra A, Sharma AK, Kumar S, Saxena AK, Pandey AK. Bauhinia variegata leaf extracts exhibit considerable antibacterial, antioxidant, and anticancer activities. Biomed Res Int. 2013;2013:915436.

18. Brusselmans K, Vrolix R, Verhoeven G, Swinnen JV. Induction of cancer cell apoptosis by flavonoids is associated with their ability to inhibit fatty acid synthase activity. J Biol Chem. 2005;280(7):5636-45.

19. Wu Y, Wang F, Zheng Q, Lu L, Yao H, Zhou C, Wu X, Zhao Y. Hepatoprotective effect of total flavonoids from Laggera alata against carbon tetrachloride-induced injury in primary cultured neonatal rat hepatocytes and in rats with hepatic damage. J Biomed Sci. 2006;13(4): 569-78.

20. Kim SM, Kang $\mathrm{K}$, Jho EH, Jung YJ, Nho CW, Um BH, Pan $\mathrm{CH}$. Hepatoprotective effect of flavonoid glycosides from Lespedeza cuneata against oxidative stress induced by tert-butyl hyperoxide. Phytother Res. 2011;25(7):1011-7

21. Gerdin B, Svensjo E. Inhibitory effect of the flavonoid O-(beta-hydroxyethyl)rutoside on increased microvascular permeability induced by various agents in rat skin. Int J Microcirc Clin Exp. 1983;2(1):39-46.

22. Cushnie TP, Lamb AJ. Antimicrobial activity of flavonoids. Int J Antimicrob Agents. 2005;26(5):343-56.

23. Middleton E Jr, Kandaswami C. Effects of flavonoids on immune and inflammatory cell functions. Biochem Pharmacol. 1992;43(6):1167-79.

24. Hunter T. Protein kinases and phosphatases: the yin and yang of protein phosphorylation and signaling. Cell. 1995;80(2):225-36.

25. Mishra A, Kumar S, Pandey AK. Scientific validation of the medicinal efficacy of Tinospora cordifolia. The Scientific World Journal. 2013;2013:292934.

26. Sun H, Liu Y, Gai Y, Geng J, Chen L, Liu H, Kang L, Tian Y, Li Y. De novo sequencing and analysis of the cranberry fruit transcriptome to identify putative genes involved in flavonoid biosynthesis transport and regulation. BMC Genomics. 2015;16(1):652.

27. Zhu J-H, Cao T-J, Dai H-F, Li H-L, Guo D, Mei W-L, Peng S-Q. De novo transcriptome characterization of Dracaena cambodiana and analysis of genes involved in flavonoid accumulation during formation of dragon's blood. Sci Rep. 2016:6:38315.

28. Chu Y, Corey DR. RNA sequencing: platform selection, experimental design and data interpretation. Nucleic Acid Ther. 2012;22(4):271-4.

29. Jain M. Next-generation sequencing technologies for gene expression profiling in plants. Brief Funct Genomics. 2012;11(1):63-70.

30. Saito K, Yonekura-Sakakibara K, Nakabayashi R, Higashi Y, Yamazaki M, Tohge T, Fernie AR. The flavonoid biosynthetic pathway in Arabidopsis: structural and genetic diversity. Plant Physiol Biochem. 2013;72:21-34.

31. Bogs J, Ebadi A, McDavid D, Robinson SP. Identification of the flavonoid hydroxylases from grapevine and their regulation during fruit development. Plant Physiol. 2006;140(1):279-91.

32. Gutha LR, Casassa LF, Harbertson JF, Naidu RA. Modulation of flavonoid biosynthetic pathway genes and anthocyanins due to virus infection in grapevine (Vitis vinifera L.) leaves. BMC Plant Biol. 2010;10:187.

33. Jin $M$, Zhang $X$, Zhao $M$, Deng $M$, Du $Y$, Zhou $Y$, Wang $S$, Tohge $T$, Fernie AR, Willmitzer $L$, et al. Integrated genomics-based mapping reveals the genetics underlying maize flavonoid biosynthesis. BMC Plant Biol. 2017:17:17.

34. Kota R, Rudd S, Facius A, Kolesov G, Thiel T, Zhang H, Stein N, Mayer K, Graner A: Snipping polymorphisms from large EST collections in barley (Hordeum vulgare L.). Mol Genet Genomics 2003, 270(1):24-33.

35. Rabausch U, Juergensen J, Ilmberger N, Böhnke S, Fischer S, Schubach B, Schulte M, Streit WR. Functional screening of metagenome and genome libraries for detection of novel flavonoid-modifying enzymes. Appl Environ Microbiol. 2013;79(15):4551-63.

36. Zhao J. Flavonoid transport mechanisms: how to go and with whom. Trends Plant Sci. 2015;20(9):576-85.

37. Zhao J, Dixon RA. The 'ins' and 'outs' of flavonoid transport. Trends Plant Sci. 2010;15(2):72-80 
38. Kitamura S, Shikazono N, Tanaka A. TRANSPARENT TESTA 19 is involved in the accumulation of both anthocyanins and proanthocyanidins in Arabidopsis. Plant J. 2004;37(1):104-14.

39. Kitamura S, Matsuda F, Tohge T, Yonekura-Sakakibara K, Yamazaki M, Saito K, Narumi I. Metabolic profiling and cytological analysis of proanthocyanidins in immature seeds of Arabidopsis thaliana flavonoid accumulation mutants. Plant J. 2010;62(4):549-59.

40. Zhao J, Huhman D, Shadle G, He XZ, Sumner LW, Tang Y, Dixon RA. MATE2 mediates vacuolar sequestration of flavonoid glycosides and glycoside malonates in Medicago truncatula. Plant Cell. 2011;23(4):1536-55.

41. Marrs KA, Alfenito MR, Lloyd AM, Walbot V. A glutathione S-transferase involved in vacuolar transfer encoded by the maize gene Bronze-2. Nature. 1995;375(6530):397-400.

42. Gomez C, Terrier N, Torregrosa L, Vialet S, Fournier-Level A, Verries C, Souquet JM, Mazauric JP, Klein M, Cheynier V, et al. Grapevine MATE-type proteins act as vacuolar $\mathrm{H}+-$ dependent acylated anthocyanin transporters. Plant Physiol. 2009;150(1):402-15.

43. Perez-Diaz R, Ryngajllo M, Perez-Diaz J, Pena-Cortes H, Casaretto JA, Gonzalez-Villanueva E, Ruiz-Lara S. VvMATE1 and VVMATE2 encode putative proanthocyanidin transporters expressed during berry development in Vitis vinifera L. Plant Cell Rep. 2014;33(7):1147-59.

44. Lepiniec L, Debeaujon I, Routaboul JM, Baudry A, Pourcel L, Nesi N, Caboche M. Genetics and biochemistry of seed flavonoids. Annu Rev Plant Biol. 2006;57:405-30

45. Zifkin M, Jin A, Ozga JA, Zaharia LI, Schernthaner JP, Gesell A, Abrams SR, Kennedy JA, Constabel CP. Gene expression and metabolite profiling of developing highbush blueberry fruit indicates transcriptional regulation of flavonoid metabolism and activation of abscisic acid metabolism. Plant Physiol. 2012;158(1):200-24.

46. Hoeren FU, Dolferus R, Wu Y, Peacock WJ, Dennis ES. Evidence for a role for AtMYB2 in the induction of the Arabidopsis alcohol dehydrogenase gene (ADH1) by low oxygen. Genetics. 1998;149(2):479-90.

47. Xie Z, Lee E, Lucas JR, Morohashi K, Li D, Murray JA, Sack FD, Grotewold E. Regulation of cell proliferation in the stomatal lineage by the Arabidopsis MYB FOUR LIPS via direct targeting of core cell cycle genes. Plant Cell. 2010; 22(7):2306-21

48. Ambawat S, Sharma P, Yadav NR, Yadav RC. MYB transcription factor genes as regulators for plant responses: an overview. Physiology and molecular biology of plants : an international journal of functional plant biology. 2013; 19(3):307-21.

49. Hichri I, Barrieu F, Bogs J, Kappel C, Delrot S, Lauvergeat V. Recent advances in the transcriptional regulation of the flavonoid biosynthetic pathway. Exp Bot. 2011;62(8):2465-83.

50. Murre C, McCaw PS, Baltimore D. A new DNA binding and dimerization motif in immunoglobulin enhancer binding, daughterless, MyoD and myc proteins. Cell. 1989;56(5):777-83.

51. Stirnimann CU, Petsalaki E, Russell RB, Muller CW. WD40 proteins propel cellular networks. Trends Biochem Sci. 2010;35(10):565-74.

52. Hasanein P, Emamjomeh A, Chenarani N, Bohlooli M. Beneficial effects of rutin in diabetes-induced deficits in acquisition learning, retention memory and pain perception in rats. Nutr Neurosci. 2018:1-12.

53. Jiang NH, Zhang GH, Zhang JJ, Shu LP, Zhang W, Long GQ, Liu T, Meng ZG, Chen JW, Yang SC. Analysis of the transcriptome of Erigeron breviscapus uncovers putative scutellarin and chlorogenic acids biosynthetic genes and genetic markers. PLoS One. 2014;9(6):e100357.

54. He M, Wang Y, Hua W, Zhang Y, Wang Z. De novo sequencing of Hypericum perforatum transcriptome to identify potential genes involved in the biosynthesis of active metabolites. PLoS One. 2012;7(7):e42081.

55. Yuan XZ. Xiaoxing; Huang, Wei; Chen, Zeming; Sun, Weihong; Li, Yanlei; Chen, Luyao; Zou, Shuangquan: study on the changes of contents of pigments of Euscaphis konishii Hayata fruit during fruit develop. Nonwood For Res. 2018;36(3):100-6 (in chinese with English Abstract).

56. Lloyd A, Brockman A, Aguirre L, Campbell A, Bean A, Cantero A, Gonzalez A. Advances in the MYB-bHLH-WD repeat (MBW) pigment regulatory model: addition of a WRKY factor and co-option of an anthocyanin MYB for Betalain regulation. Plant Cell Physiol. 2017;58(9):1431-41.

57. Nemesio-Gorriz M, Blair PB, Dalman K, Hammerbacher A, Arnerup J, Stenlid J, Mukhtar SM, Elfstrand M. Identification of Norway spruce MYB-bHLH-WDR transcription factor complex members linked to regulation of the flavonoid pathway. Front Plant Sci. 2017:8:305
58. Chiu LW, Li L. Characterization of the regulatory network of BoMYB2 in controlling anthocyanin biosynthesis in purple cauliflower. Planta. 2012; 236(4):1153-64.

59. Hancock KR, Collette V, Fraser K, Greig M, Xue H, Richardson K, Jones C, Rasmussen S. Expression of the R2R3-MYB transcription factor TaMYB14 from Trifolium arvense activates proanthocyanidin biosynthesis in the legumes Trifolium repens and Medicago sativa. Plant Physiol. 2012;159(3): 1204-20.

60. Grabherr MG, Haas BJ, Yassour M, Levin JZ, Thompson DA, Amit I, Adiconis X, Fan L, Raychowdhury R, Zeng Q, et al. Full-length transcriptome assembly from RNA-Seq data without a reference genome. Nat Biotechnol. 2011;29(7): 644-52.

61. Li B, Dewey CN. RSEM: accurate transcript quantification from RNA-Seq data with or without a reference genome. BMC bioinformatics. 2011;12:323.

62. Kanehisa M, Goto S, Kawashima S, Okuno Y, Hattori M. The KEGG resource for deciphering the genome. Nucleic Acids Res. 2004;32(Database issue): D277-80.

63. Xie C, Mao X, Huang J, Ding Y, Wu J, Dong S, Kong L, Gao G, Li CY, Wei L. KOBAS 2.0: a web server for annotation and identification of enriched pathways and diseases. Nucleic Acids Res. 2011;39(Web Server issue):W316-22.

64. Liang W, Zou X, Carballar-Lejarazú R, Wu L, Sun W, Yuan X, Wu S, Li P, Ding $H, N i$, et al. Selection and evaluation of reference genes for qRT-PCR analysis in Euscaphis konishii Hayata based on transcriptome data. Plant Methods. 2018;14(1):42.

\section{Ready to submit your research? Choose BMC and benefit from:}

- fast, convenient online submission

- thorough peer review by experienced researchers in your field

- rapid publication on acceptance

- support for research data, including large and complex data types

- gold Open Access which fosters wider collaboration and increased citations

- maximum visibility for your research: over 100M website views per year

At $\mathrm{BMC}$, research is always in progress.

Learn more biomedcentral.com/submissions 